\title{
Kevin Jones \\ Ambulatory gynaecology: a new concept in the delivery of healthcare for women
}

Received: 16 November 2005 / Accepted: 29 November 2005 / Published online: 1 July 2006

(C) Springer-Verlag Berlin / Heidelberg 2006

\begin{abstract}
Ambulatory gynaecology is a concept that combines 'one stop' clinics and 'day surgery' operations as an alternative to traditional out patient consultations and in patient surgery. This management philosophy shortens the care pathway for patients and saves resources. In order to deliver this service, gynaecologists will have to learn new skills and accept different ways of working where they are part of a multidisciplinary team rather than acting as a lone consultant skilled in every aspect of gynaecology. The concept of Ambulatory Gynaecology is in keeping with the strategic vision set in the NHS delivery plan. Therefore, a unique opportunity to trasnsform healthcare for women in the UK currently exists.
\end{abstract}

\section{Introduction}

Ambulatory gynaecology is a term more commonly used in North America than in the UK. It combines a "see and treat" management philosophy in the outpatient clinics with minimal access surgery in the day surgery unit (Fig. 1). That is, "one-stop" clinics and day surgery operations replace traditional outpatient consultations and inpatient surgery. Ambulatory gynaecology shortens the care pathway for patients and saves resources (Fig. 2).

To provide one-stop service, it is necessary to combine the clinical consultation with investigations (Table 1) in a single outpatient visit. Minimal access surgery (MAS) is a vital component of ambulatory gynaecology because it provides an alternative strategy for carrying out common gynaecological operations. Gynaecologists will have to work closely with primary care doctors and develop protocols and referral guidelines so that management in primary care is not duplicated in the hospital environment and so patients are directed to the correct multidisciplinary teams (Fig. 3). Gynaecologists will also have to learn new skills and accept different ways of working in which they

K. Jones $(\bowtie)$

Marlborough Road,

Swindon SN3 6BB, UK

e-mail: kevin.jones@smnhst.swest.nhs.uk are part of a multidisciplinary team rather than acting as lone consultants skilled in every aspect of gynaecology.

\section{The overall objectives of ambulatory gynaecology}

A large number of gynaecological conditions can now be dealt with efficiently on an outpatient basis. Whenever possible this involves one visit (i.e. "one stop"). In this way, repeat visits for investigations can be avoided, as may hospital admissions. In some instances it is possible to offer medical management of conditions previously treated surgically. If surgery is required, the aim is to utilise a MAS approach in the day surgery unit. By adopting modern advances in surgical technology and challenging traditional gynaecological practice, clinical services can be redesigned for the patients' benefit. These new approaches to gynaecology can be delivered more cost-effectively than the management strategies previously provided, which is in keeping with the NHS delivery plan [1-3].

The overall objectives of an ambulatory gynaecology management philosophy are to do the following:

1. Shorten the whole treatment process (care pathway) for patients

2. Speed up recovery and return to work for patients

3. Increase out-patient procedures

4. Increase daycase rates

5. Free up capacity on in-patient theatre lists and in hospital wards

6. Reduce the number of elective operations cancelled by the hospital

7. Reduce the overall unit cost of treatment

8. Deliver the principles of governance and controls assurance

\section{The strategic context for ambulatory gynaecology}

Primary care trusts in the UK now pay hospitals through an incentive system using a standard tariff based on national human resources group benchmarks. Work will therefore need to be undertaken locally to drive unit costs closer to 


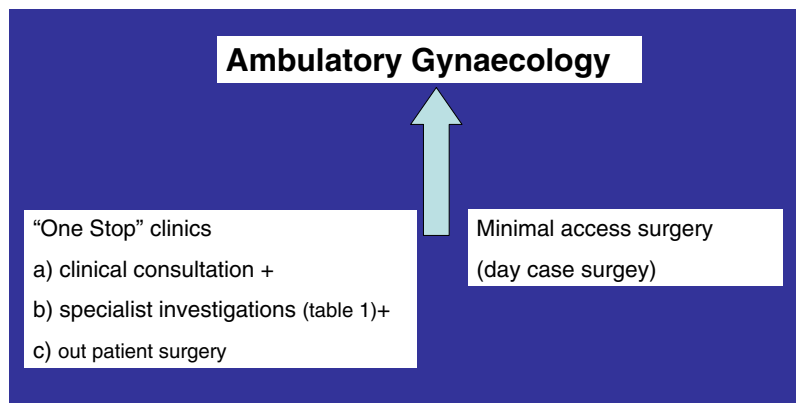

Fig. 1 The components of ambulatory gynaecology

the UK average. Hospitals are to be inspected by the Commission for Health Audit and Improvement (CHAI) and will continue to be allocated a star rating based on performance against an increasing range of performance assessment framework (PAF) indicators. CHAI will be given new powers to implement special measures if trusts do not perform at the expected level. The star rating of a trust currently affects the trust's ability to access the NHS Performance Fund, but in the future it will also affect the size of the trust's baseline allocation.

There are currently no PAF indicators directly relating to gynaecology. However, several new surgical technologies are undergoing NICE appraisal, and there has been recent media coverage that too many inappropriate hysterectomies are being done. It is likely that hysterectomy rates will be added to the indicators in the future. In addition, gynaecology activity affects the overall bed and theatre pool of the hospital and, as a result, has a direct impact on a range of key performance indicators, including trolley waits and surgical access times. The adoption of an ambulatory gynaecological management philosophy whenever possible will help individual NHS trusts achieve these objectives. Therefore, not only does this benefit individual women, but it benefits all patients using the hospital service because it makes economic sense.

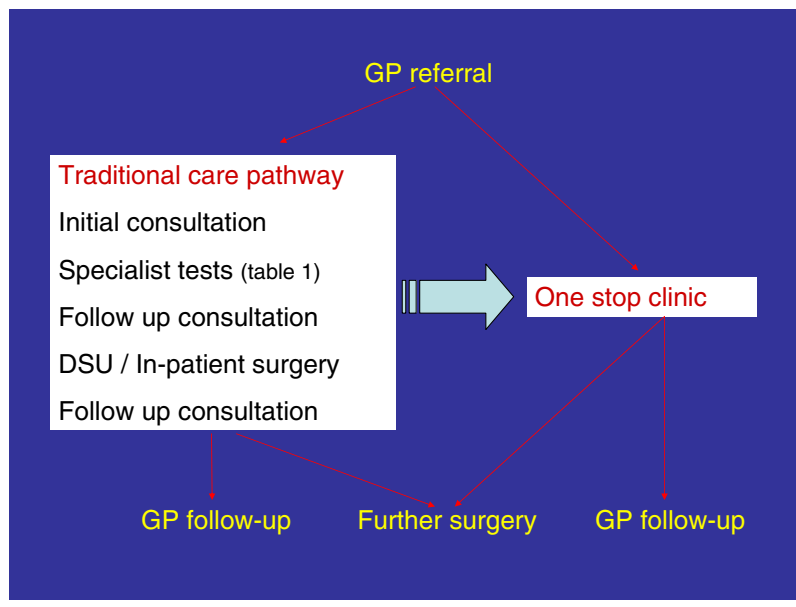

Fig. 2 Changing the care pathway for patients with gynaecological conditions
Table 1 Specific investigations/treatment for ambulatory gynaecology

\begin{tabular}{|c|c|}
\hline One-stop service & Investigation/treatment \\
\hline Abnormal uterine bleeding & $\begin{array}{l}\text { Transvaginal ultrasound scan } \\
\text { Endometrial biopsy } \\
\text { Hysteroscopy } \\
\text { (diagnostic/operative) } \\
\text { Office-based endometrial ablation }\end{array}$ \\
\hline Colposcopy & $\begin{array}{l}\text { Colposcope } \\
\text { Electrosurgical generator/cutting } \\
\text { and coagulating attachments } \\
\text { (or equivalent laser equipment) }\end{array}$ \\
\hline Fertility & $\begin{array}{l}\text { Transvaginal ultrasound scan } \\
\text { HyCoSy or transvaginal } \\
\text { hydrolaparoscopy } \\
\text { Hysteroscopy } \\
\text { Protocols for medical treatments } \\
\text { or referral to ACU }\end{array}$ \\
\hline Urogynaecology & $\begin{array}{l}\text { Transvaginal ultrasound scan } \\
\text { Urodynamic equipment } \\
\text { Flexible cystoscopy } \\
\text { Office-based continence } \\
\text { procedures }\end{array}$ \\
\hline $\begin{array}{l}\text { Early pregnancy/emergency } \\
\text { gynaecology unit }\end{array}$ & $\begin{array}{l}\text { Transvaginal ultrasound scan } \\
\text { Transvaginal ultrasound-guided } \\
\text { procedures } \\
\text { Serum beta HCG/progesterone } \\
\text { Protocols for conservative/medical } \\
\text { treatment options }\end{array}$ \\
\hline
\end{tabular}

The strategic vision set out in the NHS Plan $[1,2]$ provides the context for the development of ambulatory gynaecological services and sets out the key targets that will need to be achieved to deliver the vision. In particular, ambulatory gynaecology will help reduce waiting times by increasing overall capacity for the hospital and reducing bed occupancy and by contributing to the infrastructure

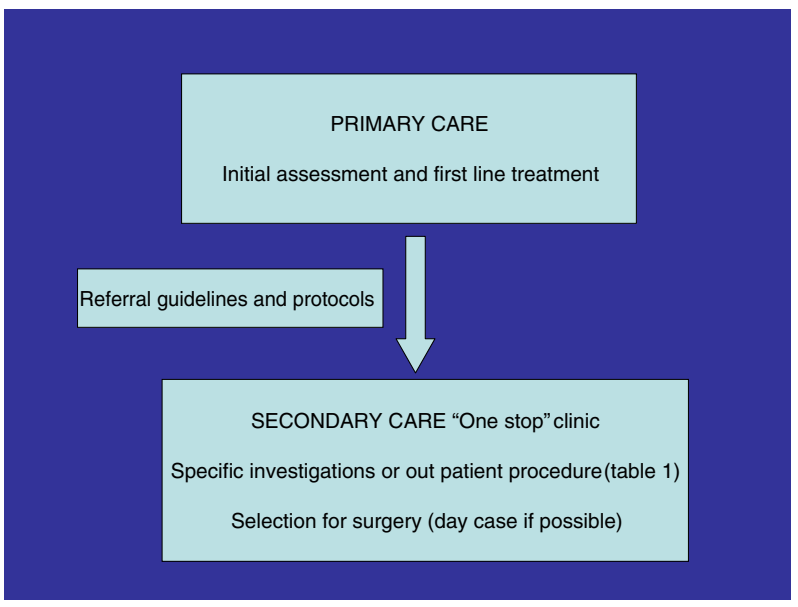

Fig. 3 Integrating primary and secondary care to deliver an ambulatory gynaecology service 
needed for services to be redesigned around the patients. Furthermore, the use of the private sector in the NHS represents a revolution in healthcare provision [4]. The key components are payment by results, patient choice, foundation hospitals, and the deliberate injection of independent treatment centres and private sector providers into the NHS. The advent of the "supplier market" presents a unique opportunity for clinicians to be entrepreneurial and innovative. Ambulatory gynaecological management strategies are an obvious way to do this.

\section{Service delivery and training}

Initiatives such as Modernising Medical Careers (MMC), the Hospital at Night project, the European Working Time Directive (EWTD), and the new "time-sensitive" contract for consultants will have a profound influence on the way hospital doctors are trained and work together in the future. These initiatives are integral parts of the government's strategy for reforming the health service $[5,6]$, which will be combined with a significant increase in consultant numbers. This should allow healthcare provision in the NHS to move from the traditional consultant-led service to a consultant-provided service.

The government's reforms of the NHS [7,8] should provide the right environment for redesigning clinical services based on one-stop management and minimal access (day-case) surgery, which are the components of an ambulatory gynaecology service. The challenge for the profession is how to restructure our units in a way that implements these reforms and utilises the new breed of specialist for the patients' benefit. The fundamental change that would have to occur to achieve this is to redefine the working relationships between consultants who would no longer act as the head of a "firm" in isolation from their colleagues. The establishment of multidisciplinary services would facilitate this [9].

\section{Establishing an ambulatory gynaecology service}

To make these changes successful, a number of changes need to occur:

1. MAS will need to be taught to all trainees. Surgeons should be able to perform operative as well as diagnostic endoscopic procedures so that a "see and treat" procedure is offered to patients. This will help reduce the number of inpatient procedures because more operations will be performed as day cases. It will also help reduce repetition of operative procedures under general anaesthetic because diagnosis and treatment can occur at the same time.

2. In the UK most gynaecology consultations involve referring the patient for an ultrasound scan. It is a pivotal investigation in gynaecology practice. Ultrasound scanning by the gynaecologist could become a routine part of the consultation rather than being delegated to radiographers and radiologists in a separate department on a separate day after a period of time on a waiting list.

The Royal College of Obstetrician and Gynaecologists (RCOG) and British Society of Gynaecological Endoscopy (BSGE) are establishing special skills modules and subspecialist training programmes to facilitate training and regulate accreditation in MAS and transvaginal ultrasound scanning TVUS.

3. Referral to hospital by family doctors should be "generic" so that patients are the responsibility of the whole department, not of an individual consultant. Under this system, individual patients are looked after by multidisciplinary teams with an appropriate special interest working together on a common clinical problem. Management strategies are evidence based and protocol driven. This helps to achieve uniformity of practice and equality of patient care.

4. To adopt a generic referral system, evidence-based/ NICE-approved protocols and referral guidelines must be in place for family doctors to follow. This will ensure that management in primary care is not duplicated in the hospital environment and that patients are directed to the correct multidisciplinary teams (Fig. 3).

5. If accredited specialists (consultants) worked together in the operating theatre or the clinic, it would facilitate acquisition of new clinical skills in order to deliver an ambulatory gynaecology service. It would also facilitate audit, patient safety, and uniformity of management [10]. The main argument put forward for maintaining the traditional system usually focuses on continuity of patient care as a result of personal patronage of individual patients. Where a multidisciplinary team approach has been adopted as part of an ambulatory gynaecology service, this is often replaced by organisational (system) continuity [11-13], e.g. the colposcopy service and the early pregnancy unit.

\section{Conclusions}

Ambulatory gynaecology combines one-stop "see and treat" clinics with day surgery management. Core skills must be applied and new working practices must be adopted in order to deliver the service that will transform healthcare for women in the UK.

\section{References}

1. Department of Health (1998) A first class service: quality in the NHS. HMSO, London

2. Department of Health (2000) The NHS plan. A plan for investment, a plan for reform. HMSO, London

3. Wanless D (2002) Securing our future health: taking a longterm view, final report. Stationary Office, London

4. Timmins N (2005) The NHS revolution: health care in the marketplace. Use of private health care in the NHS. BMJ 7525:1141-1142 
5. Pickersgill T (2001) The European working time directive for doctors in training. BMJ 323:1265-1266

6. Burke D (2002) Making the European working time directive a reality. BMJ 324:S66-S67

7. Lissauer R (2002) The future workforce. BMJ 324:S73-S74

8. BMA Health Policy and Economic Research Unit (2002) The future healthcare workforce. BMA, London

9. KD Jones (2005) Gynaecological training in a consultant delivered service: a European perspective. Obstet Gynaecol $7: 126-128$
10. Charlton R (2001) Continuing professional development (CPD) and training. BMJ 323:2-3

11. Freer SD (1999) Whither continuity of care? N Eng J Med 341:850-852

12. Manian FA (1999) Whither continuity of care? N Eng J Med 340:1362-1363

13. Krogstad U, Hofoss D, Hjortdahl P (2002) Continuity of hospital care: beyond the question of personal contact. BMJ 324:36-38 